\title{
ACTIVIDADES DA SPQ
}

\section{da Sociedade Portuguesa de Química}

COMISSÃO COORDENADORA

DO $4 .^{\circ}$ ENCONTRO ANUAL

DA SOCIEDADE PORTUGUESA DE QUÍMICA AV. ${ }^{a}$ DA REPÚBLICA, $37-4 .^{\circ}$

1000 LISBOA

\section{LOCAL}

INSTITUTO SUPERIOR TÉCNICO,

Av. Rovisco Pais, Lisboa

\section{ORGANIZAÇÃO GERAL}

O $4 .^{\circ}$ Encontro terá a duração de uma semana e será constituído por três blocos consecutivos:

Investigação, Indústria e Ensino.

É possível a inscrição em um ou mais blocos.

Haverá 6 conferências plenárias e 8 comunicaçðes convidadas. Todas as outras comunicaçðes serão apresentadas como cartazes.

\section{DATA LIMITE PARA INSCRIÇÃO}

15 de Janeiro de 1981.

As inscriçðes definitivas (ficha em anexo) e os resumos das comunicaçðes devem ser recebidos na sede da SPQ até 15 de Janeiro impreterivelmente.

\section{MEDALHA COMEMORATIVA}

Tal como em Encontros anteriores, a SPQ editará uma medalha comemorativa do $4 .^{\circ}$ Encontro Anual. Se pretende adquirir um ou mais exemplares deve preencher a inscrição em anexo (Preço aproximado: $500 \$ 00)$.

\section{COMISSÃO COORDENADORA}

A. ROMÃO DIAS (Secretário-geral da SPQ)

J.A. MARTINHO SIMÕES (Secretário Executivo do $4 .^{\circ}$ Encontro)

FRANCISCO PEDROSO (Tesoureiro)

MARIA ALZIRA A. FERREIRA (Região Sul)

A. CORREIA CARDOSO (Região Centro)

J.L. DA COSTA LIMA (Região Norte)

\section{COORDENADOR DO BLOCO INVESTIGAÇÃO}

A. PIRES DE MATOS, LNETI

COORDENADOR DO BLOCO ENSINO

VÍTOR DUARTE TEODORO, Escola Secundária de Almada

COORDENADOR DO BLOCO INDÚSTRIA

CARLOS NIETO DE CASTRO, CQE, IST

COORDENADOR DA EXPOSIÇÃO

CARLOS CRISPIM ROMÃO, CQE, IST

COORDENADOR DO PROGRAMA SOCIAL

JOÃO A. FARELEIRA, CQE, IST

\section{JANTAR DE CONVÍVIO}

Realizar-se-á um jantar de convívio no dia 8 de Abril, em local a fixar.

Se está interessado deve preencher a inscrição em anexo (Preço aproximado: 500\$00).

\section{PROGRAMA PROVISÓRIO}

DOMINGO, 5 DE ABRIL

10.00-20.00 Registo de participantes (geral)

INVESTIGAÇÃO

SEGUNDA-FEIRA, 6 DE ABRIL 
8.30-9.30 Registo de participantes 9.30-10.00 Sessão de abertura 10.00-11.00 Conferência Plenária

Autor e título a confirmar

11.00-11.30 Café

11.30-12.00 Comunicação convidada

Isomerismo rotacional: cálculos teóricos em relação com grandezas físicas

Victor M.S. Gil, Univ. Aveiro

12.10-14.30 Almoço

\subsection{0-15.30 Conferência Plenária}

The Determination of Absolute Configuration of Dissymetric Coordination Compounds by the Pseiffer effect and by Outer-Sphere Complexation

S. Kirschner, Wayne State Univ., U.S.A.

\subsection{0-16.10 Comunicação convidada}

Autor e título a confirmar

16.10-18.30 Discussão dos cartazes com os autores 19.30 Beberete

TERÇA-FEIRA, 7 DE ABRIL

\subsection{0-10.30 Conferência Plenária}

Recent Developments in the Synthesis and Biosynthesis of $\beta$-lactam Antibiotics

Jack E. Baldwin, Univ. Oxford, U.K.

10.30-11.00 Café

11.00-11.40 Comunicação convidada

Síntese de alcalóides: Da estratégia à prática

S. Prabhakar, CQE, UNL

11.40-12.20 Comunicação convidada

Estrutura e Reactividade em Química Orgânica

Carlos M.M.F. Correia, CIQ, FCUP

12.20-14.30 Almoço

14.30-15.30 Conferência Plenária

Radiation Damage of Nucleic Acids.

Mechanism of Strand Breaks Induced by $\mathrm{OH}$ Radicals

D. Schulte-Frohlind, Max Planck Institut RFA
Transferência Electrónica Fotossensibilizada

Sílvia B. Costa, CQE, IST

16.10-18.30 Discussão dos cartazes com os autores

21.00 Assembleia Geral da SPQ

QUARTA-FEIRA, 8 DE ABRIL

9.30-10.30 Conferência Plenária

Recent Developments in Gas Phase Ion Chemistry

K.R. Jennings, Univ. Warwick, U.K.

10.30-11.00 Café

11.00-11.40 Comunicação convidada

Título a confirmar (Espectroscopia Raman)

J. Teixeira Dias, FCTUC

11.40-12.20 Comunicação convidada

Dinâmica das redes cristalinas de sólidos unidimensionais. Alguns exemplos.

L.J. Alcácer, IST

12.20-14.30 Almoço

14.30-15.30 Conferência Plenária

Título a confirmar (Termoquímica)

G. Pilcher, Univ. Manchester, UK

15.30-16.10 Comunicação convidada

Estudos recentes sobre cinética e termodinâmica de reacções ionogénicas em solução

César A.N. Viana, CECUL, FCL

16.10-18.30 Discussão dos cartazes com os autores 21.00 Jantar de confraternização

\section{INDÚSTRIA}

QUINTA-FEIRA, 9 DE ABRIL

8.30- 9.00 Registo de participantes

9.00- 9.30 Sessão de abertura

9.30-10.30 Conferência Plenária

Industrial Chemical Research in the European Context

E. Sterling, ICI Europa Ltd

10.30-11.00 Café

11.00-12.45 Debate sobre Integração Europeia e seus reflexos na Indústria Química portuguesa

12.30-14.30 Almoço

14.30-15.30 Conferência Plenária 
Interacção Universidade-Indústria: Implicações Políticas

J.J.R. Fraústo da Silva, CQE, IST

15.30-16.00 Café

16.00-18.00 Debate sobre Interacção Universidade-Indústria

18.00-19.00 Discussão dos cartazes com os autores 19.00 Beberete

SEXTA-FEIRA, 10 DE ABRIL

9.30-10.00 Química Analítica na Indústria Alimentar

Conferencista a confirmar

10.00-10.30 Química Analítica na Agricultura Comunicação a cargo da Estação Agronómica Nacional, Estação Nacional de Tecnologia dos Produtos Agrários

10.30-11.00 Discussão

11.00-11.30 Café

11.30-12.30 Demonstração de equipamento

12.30-14.30 Almoço

14.30-15.00 Química Analítica na Indústria Petroquímica Comunicação a cargo da CNP

15.00-15.30 Química Analítica na Indústria Metalúrgica Extractiva

Conferencista a confirmar

15.30-16.00 Análise Química no Controlo de Produção da Siderurgia Nacional

António Ferreira de Carvalho

16.00-16.30 Café

16.30-17.00 Conclusões

Maria de Jesus Tavares, LNETI

17.00-19.00 Demonstração de Equipamento

\section{ENSINO}

\section{SEXTA-FEIRA, 10 DE ABRIL}

8.30- 9.30 Registo de participantes

9.30-10.00 Sessão de Abertura

10.00-11.00 Conferência Plenária

\section{Título a confirmar}

M. Frazer, Univ. East Anglia, U.K.

11.00-11.30 Café

11.30-12.30 Discussão dos cartazes com os autores 12.30-14.30 Almoço

14.30-16.30 Grupos de Trabalho, Filmes, Trabalhos de Laboratório

16.30-17.00 Café

17.00-19.00 Grupos de Trabalho, Filmes, Trabalhos de Laboratório

19.30 Beberete

SÁBADO, 11 DE ABRIL
Application of advances in learning theory and philosophy of science to improvement of chemistry teaching

J.D. Novak, Cornell Univ., U.S.A.

10.30-11.00 Café

11.00-12.30 Apresentação das conclusões dos Grupos de Trabalho

12.30-14.30 Almoço

14.30-17.30 Filmes, Trabalhos de Laboratório

\section{INFORMAÇÕES GERAIS}

OS CARTAZES ESTARÃO EXPOSTOS, NO DIA RESPECTIVO, DESDE AS 10 HORAS ATÉ AO FINAL DOS TRABALHOS. NO HORÁRIO MENCIONADO NO PROGRAMA O/S AUTORE/S DEVEM ESTAR JUNTO DOS SEUS CARTAZES PARA DISCUSSÃO.

OS TRABALHOS DE LABORATÓRIO REFERIDOS NO PROGRAMA ESTARÃO EM EXIBIÇÃO NOS LABORATÓRIOS DE QUÍMICA DO IST, ONDE SERÃO DEMONSTRADOS E DISCUTIDOS. SENDO TRABALHOS DE LABORATÓRIO PARA ALUNOS DO TERCIÁRIO O SEU PAPEL NESTE ENCONTRO É, ESSENCIALMENTE, O DE ACTUALIZAÇÃO. A DISCUSSÃO DA ADAPTAÇÃO, TOTAL OU PARCIAL, DE ALGUNS DELES, AO ENSINO SECUNDÁRIO, É ENCORAJADA.

DURANTE TODO O ENCONTRO HAVERÁ UMA EXPOSIÇÃO DE MATERIAL CIENTÍFICO, DIDÁCTICO E BIBLIOGRÁFICO.

A SPQ CONSIDERA EXTREMAMENTE ÚTIL QUE TODAS AS ENTIDADES, PÚBLICAS E PRIVADAS, RELACIONADAS COM A QUÍMICA EM PORTUGAL, DESDE AS QUE SE DEDICAM A ACTIVIDADE FABRIL ÀS QUE FINANCIAM A INVESTIGAÇÃO APROVEITEM ESTA OPORTUNIDADE PARA DIVULGAR A SUA ACÇÃO. PARA MAIS INFORMAČ̃̃ES CONTACTAR A COMISSÃO COORDENADORA.

DURANTE TODO O ENCONTRO O SERVICCO DE DOCUMENTAÇÃO CIENTÍFICA E TECNOLÓGICA PORÁ Ã DISPOSIÇÃO DOS PARTICIPANTES UM TERMINAL DE COMPUTADOR LIGADO A BANCOS DE DADOS. 


\section{GRUPOS DE TRABALHO DO BLOCO DE ENSINO}

1. Bancos de Objectivos
a. Curso Geral
b. Curso Complementar
c. $12 .^{\circ}$ Ano
d. $10^{\circ}$ Ano da Universidade

2. Improvisação de material de Laboratório

3. Conceito de mole

4. Segurança e Organização do Laboratório

5. Investigação de dificuldades de aprendizagem

6. Psicologia na aprendizagem da Química

7. Avaliação: projectos de teses padronizados

8. Avaliação do trabalho de Laboratório

\section{CARTAZES}

O CARTAZ É UMA FORMA EXPEDITA E EFICAZ DE TRANSMITIR UMA MENSAGEM, QUER SE TRATE DE RESULTADOS DE INVESTIGAÇÃO E/OU DE DESENVOLVIMENTO, DE INFORMAÇÃO TECNOLÓGICA OU DE OBSERVAÇÕES E OPINIÕES RESULTANTES DA EXPERIÊNCIA COMO DOCENTE. UM CARTAZ É UMA COMUNICAÇÃO E O SEU RESUMO (VER INSTRUÇÕES PARA APRESENTAÇÃO DOS RESUMOS) FIGURARÁ NA DOCUMENTAÇÃO A DISTRIBUIR. O cartaz deve ter as dimensøes de $\mathbf{2} \mathbf{m}$ de largura por $\mathbf{1 , 2 0} \mathbf{~ m}$ de altura. DEVE SER ESCRITO DE FORMA CLARA E O SEU TÍTULO DEVE SER LEGÍVEL A 3 M DE DISTÂNCIA. PODE CONTER TEXTO, DESENHOS,
FOTOGRAFIAS, AMOSTRAS DE COMPOSTOS, MODELOS, ETC. O USO DE VÁRIAS CORES E A INCLUSÃO DA/S FOTOGRAFIA/S DO/S AUTOR/ES É ENCORAJADO.

OS AUTORES DEVEM TRAZER O CARTAZ PARA O ENCONTRO E AFIXÁ-LO E RETIRÁ-LO, EM LOCAL INDICADO, NO HORÁRIO REFERIDO NO PROGRAMA.

NO BLOCO DE ENSINO SERÃO ADMITIDOS, COMO ALTERNATIVAS A CARTAZES, E COM O MESMO HORÁRIO DE EXIBIÇÃO, EXPERIÊNCIAS DE LABORATÓRIO, COLECÇÕ̃ES DE ACETATOS, COLECCÕES DE SLIDES, ETC. A COMISSÄO COOORDENẢDORA PROCURARA ÄRRANJAR AS FACILIDADES NECESSÁRIAS PARA ESTAS ALTERNATIVAS, DEVENDO, PARA ISSO, OS AUTORES EXPLICITÁ-LAS NOS BOLETINS DE INSCRIÇÃO.

\section{DIVISÃO DE NOMENCLATURA}

No dia 10 de Abril, 6. ${ }^{\text {a }}$-feira, às 14.30 h, haverá uma reunião entre os interessados em problemas de nomenclatura química.

\section{MARCAÇÃO DE ALOJAMENTO}

A Comissão Coordenadora poderá encarregar-se da marcação de alojamento.

Na Ficha de Inscrição deverá ser indicada uma ordem de preferência. Se desejar pode indicar quaisquer outros Hotéis que não figurem na lista.

\begin{tabular}{|c|c|c|c|c|c|c|}
\hline $\begin{array}{c}\text { HOTÉIS } \\
\text { E } \\
\text { RESIDENCIAIS }\end{array}$ & SINGLE & DUPLO & TRIPLO & QUADRUPLO & MORADA & TELEF. \\
\hline $\begin{array}{l}\text { HOTEL } \\
\text { D. MANUEL I } \\
* * * *\end{array}$ & $1750 \$$ & $2250 \$$ & - & - & $\begin{array}{c}\text { Av. Duque } \\
\text { D'ÁvILA, } 187\end{array}$ & 561410 \\
\hline $\begin{array}{l}\text { HOTEL } \\
\text { ROMA } \\
* * *\end{array}$ & $975 \$$ & $1450 \$$ & $2062 S$ & $2500 \$$ & Av. de Roma, 33 & 767761 \\
\hline $\begin{array}{l}\text { HOTEL } \\
\text { PRINCIPE } \\
* * *\end{array}$ & $1200 \$$ & $1600 \$$ & - & - & $\begin{array}{l}\text { Av. Duque } \\
\text { D'Ávila, } 201\end{array}$ & 536151 \\
\hline HOTEL EDUARDO VII & $\begin{array}{l}1105 \$ \\
1305 \$\end{array}$ & $\begin{array}{l}1400 \$ \\
1400 \$\end{array}$ & - & - & $\begin{array}{l}\text { Av. Fontes Pereira } \\
\text { de Melo, } 5\end{array}$ & 530141 \\
\hline $\begin{array}{l}\text { PENSÃO RESIDENCIAL } \\
\text { NAZARETH } \\
* * * *\end{array}$ & $720 \$$ & $1050 \$$ & - & - & $\begin{array}{l}\text { Av. António Augusto } \\
\text { de Aguiar, 25-4. }\end{array}$ & 542016 \\
\hline RESIDÊNCIA AMÉRICA & $700 s$ & $900 s$ & $1150 \$$ & - & Rua Tomás Ribeiro, 47 & 531178 \\
\hline RESIDÊNCIA CANADÁ & $510 \$$ & $800 \$$ & 1075 & - & $\begin{array}{l}\text { Av. Defensores } \\
\text { de Chaves, 35-1. }\end{array}$ & 538159 \\
\hline $\begin{array}{l}\text { PENSÃO RESIDENCIAL } \\
\text { DO SUL }{ }^{* * *}\end{array}$ & $\ddot{350 \text { s }}$ & $\because \bullet$ & - & - & $\bullet$ & 847259 \\
\hline
\end{tabular}

- - PREÇOS REFERENTES A OUTUBRO DE 1980 (SUJEITOS A AUMENTO)

•- FILIAIS EM: - AV. ${ }^{a}$ ALMIRANTE REIS, 28-1. 